\title{
Contactless Detection of Facial Signs Related to Stress: A Preliminary Study
}

\author{
Dimitris Manousos ${ }^{1}$, Galateia Iatraki ${ }^{1}$, Eirini Christinaki ${ }^{1}$, Matthew Pediaditis ${ }^{1}$, Franco Chiarugi ${ }^{1}$, Manolis \\ Tsiknakis $^{1,2}$, Kostas Marias $^{1}$ \\ ${ }^{1}$ Computational Medicine Laboratory, Institute of Computer Science, Foundation for Research and Technology - Hellas, \\ Heraklion, Crete, Greece \\ ${ }^{2}$ Department of Informatics Engineering, Technological Educational Institute of Crete, \\ Heraklion, Crete, Greece \\ \{mandim, giatraki, echrist, mped, chiarugi, tsiknaki, kmarias\}@ics.forth.gr
}

\begin{abstract}
This paper presents a contactless methodology for detecting facial signs related to stress. A brief literature review shows that there are specific facial signs that are related to stressful conditions. A methodology based on computer vision techniques applied to color videos in order to extract facial signs such as movement (head), eyebrow lowering and raising, and blink rate is presented. Various facial features are investigated and are quantitatively evaluated through a frame-to-frame analysis. Preliminary results with few volunteers reveal a direct correlation of the selected facial signs with stress conditions. These initial results confirm our hypothesis regarding the possibility of assessing stress from facial expressions. However, these preliminary results should be quantitatively verified on a more comprehensive dataset containing a significant number of subjects in order to validate the algorithm results.
\end{abstract}

Keywords- stress; contactless detection; face detection; facial signs; head movement; eyebrows movement; blink rate.

\section{INTRODUCTION}

Stress is the psychosomatic response to the stressors and is often induced, as a side effect, by accelerated life rhythms. A stressor is the stimulus or event which causes the stress. Stressors are perceived by the human body as threats, with the consequent mobilization of all body resources for fighting or fleeing from these threats. Stress is considered to be itself a risk factor for cardiovascular diseases [1], but it must be highlighted that high levels of stress may increase other related risk factors such as high cholesterol or high blood pressure. Therefore, the detection of stress in its early stages turns to be of great significance, especially if achieved in a contactless way without the excessive use of sensors or other monitoring equipment, which may cause extra stress to the individual [2]. Moreover, computer vision offers a way to assess a person's behavior similarly to that of a human observer, but in a more quantitative way. Such approaches have been reported in other areas of health monitoring and assessment (e.g. epilepsy [3]).

This paper describes an approach based on the detection of three facial signs for the recognition of stress. These signs consist of head motion, eyebrow lowering and raising, and blink rate. Algorithms for the detection of these facial signs have been designed and implemented and initial tests of the developed algorithms have been performed on videos of two volunteers, being part of the SEMEOTICONS project, collected under different external stimuli. Preliminary results show discernible quantitative differences between the facial signs extracted from videos related to the subject in a relaxing phase and videos related to the same subject under stress.

The remaining of this paper is structured as follows: Section II presents a brief literature review of facial signs related to stress, the overall methodology, as well as the description of the algorithms for the extraction of the three aforementioned facial signs. Section III documents the attained preliminary results and finally, a discussion and the conclusions are reported in Section IV.

\section{METHODOLOGY}

\section{A. Facial signs related to stress}

Facial signs related to stress can be recognized in the head and face as a whole or in specific local regions of the face, such as the eyelids and eyebrows, the eyeballs, the pupil, the mouth and the lips. The facial signs obtained from the head are: movement (head) [4], tilt frequency, movement speed and head orientation [5]. Other facial signs in the face as a whole are associated to the skin color (frequent blushing, reddening or pallor [6], [7]) or to the muscle hyperactivity. Analyzing local regions of the face, if we consider the eyelids, the most important facial signs of stress are the blink rate, speed and duration, amplitude, twitching and response. Considering the eyeballs, saccadic eye movements and gaze spatial distribution are facial signs also related to stress [8]. Finally, raising and lowering of eyebrows [9], pupil diameter, mouth jaw clenching, lip trembling and stiffening are related to stress as well [10], [11], [12].

\section{B. Approach for extraction of facial signs}

There are various methods reported in the literature for detecting facial signs related to stress, by using computer vision. Most of them can be applied either to the whole face or head (holistic approach) or to specific regions of interest (ROI) like the mouth, nose or eyes (local approach). Although the holistic approach provides a complete picture a facial expression, in cases where facial signs are located in specific regions, a local approach gives more detailed and distinguishable information. 
Facial sign extraction methods can be categorized in: muscle/geometric based, model/appearance based, motion based and their combination as hybrid methods.

Our methodology detects and estimates three very relevant facial signs from the aforementioned ones in Section II.A by processing two-dimensional (2D) recorded color videos with a predefined resolution and frame rate. The selected facial signs are head movement, eyebrows lowering and raising, and blink rate.

Three different methods that measure these facial signs have been implemented following a common workflow, which takes as input the recorded video. Face detection is explicitly performed as a starting point through the Viola-Jones [13] algorithm. This step is applied for the eyebrow and head motion, but not for the blink rate, where an Active Appearance Model (AAM), which does not require explicitly this preliminary step, is used. In a second step, specific ROIs are selected within the area of the face that are related to each of the local facial signs. The final step is the facial feature extraction in which the facial signs are measured. All methods were implemented with MATLAB.

The methods were tested on color videos with a resolution of $526 \times 696$ pixels at 50 frames per second (fps) in a task-based procedure of about $7 \mathrm{~min}$ performed on two volunteers participating in the SEMEOTICONS project. The volunteers were recorded while watching three different 2-min length videos (a relaxing video, a video from heights/adventure film and a video with psychological pressure), with $30 \mathrm{sec}$ pause inbetween, in order to reveal their facial expressions related to relax or stress. The subjects were seated at a distance of about $0.5 \mathrm{~m}$ from the camera in condition of diffuse illumination and with their attention focused on a screen, located in front of them, where the films were shown.

\section{Movement (head)}

This algorithm measures the head motion in terms of horizontal and vertical deviation of specific reference points between consecutive frames. After the face detection, a local region of interest (ROI) has to be selected with absence or very low local movements in order to measure optimally the head motion. In fact, the algorithm needs to monitor the head movement and to discard movements that are related to other factors, such as mouth movements, eye blinks, etc. Our assumption is that the region between the eyes and mouth is the most appropriate region since there is absence of local movements. After the selection of this ROI, the next step of the algorithm is the choice of specific reference points that are located at the 4 edges of the ROI. Then, a tracker based on Lukas's and Kanade's [14] optical flow is applied for tracking their position frame by frame. A motion filter is used in order to discard erratic trajectories and unstable points, in case the reference points are unstable and produce irregular trajectories. To retain the most stable reference points, the maximum distance traveled by each point between consecutive frames is calculated and points with a distance exceeding the mode of the distribution are discarded. Finally, the reference point trajectories are analyzed in order to produce three different signals related to the frame by frame movements: scalarly in horizontal and vertical, and also vectorially through the deviation magnitude.

\section{Eyebrow movement}

This algorithm measures the movement of eyebrow points that typically characterizes the motion of the entire eyebrow. After face detection, a ROI that includes eyes and eyebrows is defined. Our goal is to use eye corners as reference points for tracking eyebrow movements. The next step after ROI definition is to convert the image into grayscale and introduce contrast enhancement using histogram equalization. Afterwards, image is converted to binary and morphological operations are applied in order to remove small objects and to smooth features through morphological closing.

Our method for eyebrow segmentation is based on the observation that facial features differ from the rest of the face due to their low brightness [15]. Hence, the algorithm searches for strong local peaks in horizontal and vertical projections of the binary image. Our hypothesis is that eyebrows generate a maximum in the horizontal projection and the left and right boundaries can be determined by peaks in the vertical projection.

The initial algorithm for the selection of the specific points (around peak values) on the eyebrows was adjusted and refined through testing on a subset of additional videos recorded on volunteer participants to the project, in order to enhance its reliability. After the automatic selection of the most suitable points, the algorithm calculates the change in distance between each eyebrow point and the corresponding reference eye corner point.

This information can be used for the evaluation of the eyebrow lowering (decrease of the distance) and eyebrow raising (increase of the distance).

\section{E. Blink rate}

One of the most often reported parameters that fluctuates among different stress levels is the blink rate [16], [17], [18]. In this study it is extracted using Active Appearance Models [19]. More specifically, the eye area is segmented and eyelid perimeter is designated with specific landmarks (6 landmark points used for each eye). Then the average distance between the upper and lower eye points is calculated. This signal represents the eye opening. After visual inspection of the resulted signal, a threshold was established ( 5 pixels distance below the average distance of points). Since an eye blink lasts from 100 to $400 \mathrm{~ms}$, at least 5 consecutive values $(100 \mathrm{~ms})$ need to be below that threshold in order for an eye blink to be detected.

\section{RESULTS}

As already mentioned in Section II, the above algorithms have been tested in a preliminary manner with videos that have been recorded from two volunteers while watching three different short films: one relaxing or normal and two apparently stressful.

Regarding the head movement, we considered the mean value, the standard deviation and the median value of the generated time series of the X-Y motion as well as the vector magnitude in the relaxed and stressed states. As shown in TABLE I, the first subject had more intense movements during 
the stressed condition than during the relaxed phase. Regarding the second subject the head motion values showed no major differences between any states. However, the first subject reported that the most stressful film was the heights/adventure film (Stress 1) and for this one the standard deviation is significantly increased compared to the relaxed state. The second subject reported that the most stressful film was the one with psychological pressure (Stress 2) and still for this subject the standard deviation of the movements measured during the vision of this film results increased compared to the relaxed state.

The limited number of subjects analyzed do not allow to draw definite conclusions on specific stress indicators. However, there is a clear increase in the involuntary head movements in the stress state compared to the relaxed one and that should be explored as potential indicator for stress assessment.

TABLE I. HEAD MOTION RESULTS

\begin{tabular}{|c|c|c|c|c|}
\hline & & \multicolumn{3}{|c|}{ Subject 1} \\
\hline & & Mean & Median & $S t d$ \\
\hline \multirow{3}{*}{$X$} & Relaxing $^{1}$ & 0.040 & 0.027 & 0.059 \\
\hline & Stress $1^{2}$ & 0.080 & 0.040 & 0.114 \\
\hline & Stress $2^{3}$ & 0.067 & 0.049 & 0.065 \\
\hline \multirow{3}{*}{ Y } & Relaxing $^{1}$ & 0.057 & 0.044 & 0.053 \\
\hline & Stress $1^{2}$ & 0.122 & 0.076 & 0.638 \\
\hline & Stress $2^{3}$ & 0.057 & 0.042 & 0.053 \\
\hline \multirow{5}{*}{ Mags } & Relaxing $^{1}$ & 0.106 & 0.095 & 0.081 \\
\hline & Stress $1^{2}$ & 0.164 & 0.119 & 0.644 \\
\hline & Stress $2^{3}$ & 0.115 & 0.099 & 0.078 \\
\hline & & \multicolumn{3}{|c|}{ Subject 2} \\
\hline & & Mean & Median & Std \\
\hline \multirow{3}{*}{ X } & Relaxing $^{1}$ & 0.159 & 0.196 & 0.179 \\
\hline & Stress $1^{2}$ & 0.082 & 0.058 & 0.090 \\
\hline & Stress $2^{3}$ & 0.151 & 0.095 & 0.205 \\
\hline \multirow{3}{*}{ Y } & Relaxing $^{1}$ & 0.156 & 0.107 & 0.172 \\
\hline & Stress $1^{2}$ & 0.064 & 0.044 & 0.067 \\
\hline & Stress $2^{3}$ & 0.104 & 0.060 & 0.212 \\
\hline \multirow{3}{*}{ Mags } & Relaxing $^{1}$ & 0.248 & 0.204 & 0.222 \\
\hline & Stress $1^{2}$ & 0.222 & 0.121 & 0.111 \\
\hline & Stress $2^{3}$ & 0.244 & 0.179 & 0.281 \\
\hline
\end{tabular}

Considering the eyebrow movement we compared the mean value, standard deviation and variation among the states. In TABLE II the results for the movement of the left outer eyebrow point are shown. For both subjects we observe that all values are higher in stressed states and a higher variance is even more evident in stressed states.

We cannot draw conclusions on specific stress indicators, given the limited number of cases, but the differences between the relaxed and the stress states are relevant, suggesting that standard deviation and variance might be considered as candidate indicator for stress estimation.

TABLE II. EYEBROW MOVEMENT RESULTS

\begin{tabular}{|l|c|c|c|c|}
\hline \multirow{2}{*}{} & \multicolumn{4}{|c|}{ Subject 1 } \\
\cline { 2 - 5 } & Mean & Median & Std & Var \\
\hline Relaxing $^{1}$ & 57.045 & 56.592 & 2.196 & 5.273 \\
\hline Stress 1 $^{2}$ & 64.806 & 64.613 & 2.609 & 6.805 \\
\hline Stress 2 & 55.211 & 55.869 & 3.228 & 10.420 \\
\hline & Mean & Subject 2 \\
\cline { 2 - 5 } & 57.309 & 57.216 & 1.778 & 3.161 \\
\hline Relaxing $^{3}$ & 57.706 & 57.337 & 1.907 & 3.638 \\
\hline Stress 1 & & Std & Var \\
\hline Stress 2 & 58.121 & 58.205 & 1.795 & 3.221 \\
\hline
\end{tabular}

The results of blink rate are presented in TABLE III, where the number of eye blinks during the entire recording have been reported for both subjects and for all their videos.

These results show that there are differences among the relaxed and stressed states. As we can observe there is an increased blink rate in Stress 2 state when compared to the relaxed state. The same observation occurs on the $2^{\text {nd }}$ subject, but we cannot obtain any preliminary indication based on the blink rate evaluation in case of Stress 1.

TABLE III. BLINK RATE RESULTS

\begin{tabular}{|l|c|c|c|c|}
\hline & Subject 1 & Subject 2 & \\
\cline { 2 - 5 } & Eye blink & Eye blink & Mean & Std \\
\hline Relaxing $^{1}$ & 31 & 17 & 24 & 9.90 \\
\hline Stress 1 & 19 & 19 & 19 & 0.00 \\
\hline Stress 2 & 41 & 29 & 35 & 8.49 \\
\hline \multicolumn{4}{|c|}{$\begin{array}{c}\text { 2. Watching a video from heights/adventure film } \\
\text { 3. Watching a video with psychological pressure }\end{array}$}
\end{tabular}

\section{DISCUSSION AND CONCLUSIONS}

This paper presents a preliminary study on specific head and facial sign measures that could be exploited in order to estimate stress in a contactless way. This study conducted a literature review in order to report the most relevant facial signs related to stress and the corresponding methodologies that can be used for the estimation and measurement of such facial signs. Following this review, three methods for the evaluation of these facial signs related to stress (i.e. head motion, blink rate and 
eyebrow movement) from 2D videos have been implemented. The methods were preliminarily tested on two volunteers.

Some potential indicators for stress assessment have been obtained from each of the implemented algorithms. However, given the limited number of subjects, an extensive test on an annotated dataset has been planned and will be performed in order to validate the results for the reported algorithms and to draw solid conclusions after comparison with other published methods. In fact, the trend was not completely clear, suggesting that the way each person reacts to stress or perceive stress is different. This extensive test might also suggests additional tuning or calibration of the implemented algorithms for the achievement of sound outcomes. It cannot be ruled out that the combination of the results of the algorithms might produce strongest indicators of a potential stress state.

\section{ACKNOWLEDGMENT}

This work was performed in the framework of the FP7 Specific Targeted REsearch Project SEMEOTICONS (SEMEiotic Oriented Technology for Individual's CardiOmetabolic risk self-assessmeNt and Self-monitoring) partially funded by the European Commission under Grant Agreement 611516.

The authors would like to thank Dr. G. Giannakakis for data preparation/preprocessing and technical support.

\section{REFERENCES}

[1] F. Chiarugi, E. Christinaki, S. Colantonio, G. Coppini, P. Marraccini, M. Pediaditis, O. Salvetti and M. Tsiknakis, "A virtual individual's model based on facial expression analysis: A non-intrusive approach for wellbeing monitoring and self-management," in Bioinformatics and Bioengineering (BIBE), 2013 IEEE 13th International Conference on, Chania, Greece, 2013.

[2] F. Chiarugi, G. Iatraki, E. Christinaki, D. Manousos, G. Giannakakis, M. Pediaditis, A. Pampouchidou, K. Marias and M. N. Tsiknakis, "Facial signs and psycho-physical status estimation for well-being assessment," in The International Conference on Health Informatics (HEALTHINF 2014), Angers, France, 2014.

[3] M. Pediaditis, M. Tsiknakis and N. Leitgeb, "Vision-based motion detection, analysis and recognition of epileptic seizures - A systematic review," Computer methods and programs in biomedicine, vol. 108, no. 3, pp. 1133-1148, 2012.

[4] D. F. Dinges, R. L. Rider, J. Dorrian, E. L. McGlinchey, N. L. Rogers, Z. Cizman, S. K. Goldenstein, C. Vogler, S. Venkataraman and D. N. Metaxas, "Optical computer recognition of facial expressions associated with stress induced by performance demands," Aviation, Space, and Environmental Medicine, vol. 76, no. 6 Suppl, pp. B172B182, 2005.
[5] M. Kutila, Methods for machine vision based driver monitoring applications, PhD thesis, VTT Technical Research Centre of Finland, 2006.

[6] Stress.org, "50 common signs and symptoms of stress - the American Institute of Stress," [Online]. Available: http://www.stress.org/stresseffects/.

[7] P. D. Drummond, "Correlates of facial flushing and pallor in angerprovoking situations," Personality and Individual Differences, vol. 23, no. 4, pp. 575-582, 1997.

[8] N. Sharma and T. Gedeon, "Hybrid genetic algorithms for stress recognition in reading," in Evolutionary Computation, Machine Learning and Data Mining in Bioinformatics, Lecture Notes in Computer Science, vol. 7833, 2013, pp. 117-128.

[9] W. Liao, W. Zhang, Z. Zhu and Q. Ji, "A real-time human stress monitoring system using dynamic Bayesian network," in The IEEE Computer Society Conference on Computer Vision and Pattern Recognition (CVPR'05), San Diego, CA, USA, 2005.

[10] P. Ren, A. Barreto, Y. Gao and M. Adjouadi, "Affective assessment by digital processing of the pupil diameter," IEEE Transactions on Affective Computing, vol. 4, no. 1, pp. 2-14, 2013.

[11] F. Onorati, R. Barbieri, M. Mauri, V. Russo and L. Mainardi, "Reconstruction and analysis of the pupil dilation signal: Application to a psychophysiological affective protocol," in The Annual International Conference of the IEEE Engineering in Medicine and Biology Society (EMBC 2013), Osaka, Japan, 2013.

[12] L. J. M. Rothkrantz, R. J. van Vark and D. Datcu, "Multi-medial stress assessment," in The IEEE International Conference on Systems, Man and Cybernetics (SMC 2004), The Hague, The Netherlands, 2004.

[13] P. Viola and M. J. Jones, "Robust real-time face detection," International Journal of Computer Vision, vol. 57, no. 2, pp. 137-154, 2004.

[14] B. D. Lucas and T. Kanade, "An iterative image registration technique with an application to stereo vision," in The 7th International Joint Conference on Artificial intelligence (IJCAI'81), Vancouver, BC, Canada, 1981.

[15] K. Sobottka and I. Pitas, "Extraction of facial regions and features using color and shape information," in The 13th International Conference on Pattern Recognition (ICPR 1996), Vienna, Austria, 1996.

[16] J. A. Harringan and D. M. O'Connell, "How do you look when feeling anxious? Facial displays of anxiety," Personality and Individual Differences, vol. 21, no. 2, pp. 205-212, 1996.

[17] M. Argyle and M. Cook, Gaze and mutual gaze, New York, NY, USA: Cambridge University Press, 1976.

[18] C. S. Harris, R. I. Thackray and R. W. Shoenberger, "Blink rate as a function of induced muscular tension and manifest anxiety," Perceptual and Motor Skills, vol. 22, no. 1, pp. 155-160, 1966.

[19] T. F. Cootes, G. J. Edwards and C. J. Taylor, "Active appearance models," IEEE Transactions on Pattern Analysis and Machine Intelligence, vol. 23, no. 6, pp. 681-685, 2001. 